\title{
Genome-wide association studies (GWAS) to provide insights into the complex interplay of nuclear receptor transcriptional networks and their contribution to the maintenance of homeostasis
}

\author{
Thanasis Mitsis ${ }^{1}$, Dimitrios Vlachakis ${ }^{1,2,3}$, George P. Chrousos ${ }^{2,3}$, Elias Eliopoulos ${ }^{1}$ \\ ${ }^{1}$ Laboratory of Genetics, Department of Biotechnology, School of Applied Biology \& Biotechnology, Agricultural University of \\ Athens, Athens, Greece \\ ${ }^{2}$ University Research Institute of Maternal and Child Health \& Precision Medicine, and UNESCO Chair on Adolescent Health Care, \\ National and Kapodistrian University of Athens, "Aghia Sophia" Children's Hospital, Athens, Greece \\ ${ }^{3}$ Division of Endocrinology and Metabolism, Center of Clinical, Experimental Surgery and Translational Research, Biomedical \\ Research Foundation of the Academy of Athens, Athens, Greece \\ Competing interests: TM none; DV none; GPC none; EE none
}

Genome-wide association studies (GWAS) have become an essential tool in exploring the relationship between common sequence variation sites and specific traits (Tam et al., 2019). A protein family that provides ample research material for GWAS are nuclear receptors. These receptors comprise one of the largest groups of transcriptional factors and regulate the activity of a wide range of biological processes (Weikum et al., 2018). A biological system that seems to be heavily reliant on nuclear receptors' activity is homeostasis. Homeostasis can be described as the inner equilibrium, both physical and chemical, required for proper organism function. Homeostasis can be threatened by internal or external unforeseen stimuli called stressors, and as such, organisms have developed a complex mechanism that copes with such threats and acts to maintain homeostasis called the stress response system (Chrousos, 2009). This research uses the glucocorticoid receptor (GR), a vital mediator of the stress response system (Nicolaides et al., 2015), and a heavily researched nuclear receptor as the basis of a GWAS research on the interplay between nuclear receptors and homeostasis. Specifically, a comprehensive list of epigenetic factors, receptor cofactors, and enzymes that interact with GR was constructed in an effort to create a concise network of the various biological functions this receptor partakes. This network, plus the remaining nuclear receptors found in humans was studied with a large genomic dataset. The results are expected to provide insight into the interplay of nuclear receptor transcriptional networks and their contribution to homeostasis maintenance.

\section{References}

1. Chrousos GP (2009) Stress and disorders of the stress system. Nature Reviews Endocrinology 5(7), 374-81. http://dx.doi. org/10.1038/nrendo.2009.106

2. Nicolaides NC, Kyratzi E, Lamprokostopoulou A, Chrousos GP, Charmandari E (2015) Stress, the stress system and the role of glucocorticoids. Neuroimmunomodulation 22(1-2), 6-19. http:// dx.doi.org/10.1159/000362736

3. Tam V, Patel N, Turcotte M, Bossé Y, Paré G et al. (2019) Benefits and limitations of genome-wide association studies. Nature Reviews Genetics 20(8), 467-484. http://dx.doi.org/10.1038/. s41576-019-0127-1

4. Weikum ER, Liu X, Ortlund EA (2018) The nuclear receptor superfamily: A structural perspective. Protein Sci. 27(11), 187692. http://dx.doi.org/10.1002/pro.3496 